\title{
Poor convexity and Nash equilibria in games
}

\author{
Tadeusz Radzik
}

Accepted: 21 April 2013 / Published online: 15 May 2013

(C) The Author(s) 2013. This article is published with open access at Springerlink.com

\begin{abstract}
This paper considers two-person non-zero-sum games on the unit square with payoff functions having a new property called poor convexity. This property describes "something between" the classical convexity and quasi-convexity. It is proved that various types of such games have Nash equilibria with a very simple structure, consisting of the players' mixed strategies with at most two-element supports. Since poor convexity is a basic notion in the paper, also a theory of poorly convex functions is also developed.
\end{abstract}

Keywords Nash equilibrium · Two-person sum game · Non-zero sum game · Two-point strategy $\cdot$ Poor convexity

\section{Introduction}

The problem of existence of the solution introduced by Nash (1951) for noncooperative games, is widely studied in the literature. In particular, many of such results have been obtained under assumptions related to different types of concavity or convexity of payoff functions, possibly discontinuous. We can mention here several recent papers like Bich (2009), Carmona (2009), Carmona (2010), McClendon (1986) and Połowczuk et al. (2007).

The aim of the paper is to study the problem of the existence of "simple" Nash equilibria formed by so-called two-point strategies, that is such mixed strategies whose supports consist of at most two of players' pure strategies. We examine this problem for two-person non-zero-sum games on the unit square with payoff functions possessing

T. Radzik $(\bowtie)$

Institute of Mathematics and Computer Science, Wrocław University of Technology,

Wybrzeże Wyspiańskiego 27, 50-370 Wrocław, Poland

e-mail: tadeusz.radzik@pwr.wroc.pl 
some new properties called poor concavity and poor convexity. It is worth mentioning that several results of this type were found both for finite games (Połowczuk 2006, 2003; Połowczuk et al. 2007; Radzik 2000) and for games on the unit square (Parthasarathy and Raghavan 1975; Radzik 1991, 1993) with convex and concave payoff functions.

Five new "existence theorems" are proposed for games in which players' payoff functions are poorly concave or poorly convex in various configurations. This new notion of poor convexity is a natural generalization and extension of the classical convexity. In the paper, we develop the theory of poorly convex functions, using these results further in the proofs of the theorems.

The organization of the paper is as follows. Section 2 is devoted to preliminary definitions and some background results which are a starting point for our study. In Sect. 3 we introduce the new basic notions of poorly convex functions and discuss them. In Sect. 4 we present our five main results about the existence of Nash equilibria in games. Poorly concave functions and their properties are studied in Sects. 5 and 6, while Sect. 7 contains the proofs of the theorems.

\section{Preliminary results}

In this section we introduce preliminary notions and quote three basic results from the literature (Theorems A-C) which are an inspiration for our considerations in the paper.

Throughout this paper we consider only two-person non-zero-sum games on the unit square, that is the games with normal form

$$
\Gamma=\left\langle\{1,2\},\left\{X_{1}, X_{2}\right\},\left\{F_{1}, F_{2}\right\}\right\rangle
$$

where

1. $\{1,2\}$ is the set of two players;

2. for $i=1,2, X_{i}=[0,1]$ is the interval space of pure strategies $x_{i}$ of Player $i$.

3. $F_{1}\left(x_{1}, x_{2}\right)$ and $F_{2}\left(x_{1}, x_{2}\right)$ are bounded functions on $X_{1} \times X_{2}$, and for $i=$ $1,2, F_{i}\left(x_{1}, x_{2}\right)$ describes the payoff function of Player $i$, in the situation when Players 1 and 2 use their pure strategies $x_{1}$ and $x_{2}$, respectively.

A mixed strategy for Player $i$ is any probability measure $\mu_{i}$ on $X_{i}, i=1,2$. We will also write $F_{i}\left(\mu_{1}, \mu_{2}\right)=\iint F_{i}\left(x_{1}, x_{2}\right) d \mu_{1}\left(x_{1}\right) d \mu_{2}\left(x_{2}\right)$ for $i=1,2$. So $F_{i}\left(\mu_{1}, \mu_{2}\right)$ describes the expected payoff of Player $i$ when Players 1 and 2 use their mixed strategies $\mu_{1}$ and $\mu_{2}$, respectively, and the vector $\left(F_{1}\left(\mu_{1}, \mu_{2}\right), F_{2}\left(\mu_{1}, \mu_{2}\right)\right)$ is the payoff vector corresponding to the pair $\left(\mu_{1}, \mu_{2}\right)$.

A (mixed) Nash equilibrium in game $\Gamma$ is any pair $\left(\mu_{1}^{*}, \mu_{2}^{*}\right)$ of players' mixed strategies that satisfy the inequalities

$$
F_{1}\left(\mu_{1}^{*}, \mu_{2}^{*}\right) \geq F_{1}\left(\mu_{1}, \mu_{2}^{*}\right) \quad \text { and } \quad F_{2}\left(\mu_{1}^{*}, \mu_{2}^{*}\right) \geq F_{2}\left(\mu_{1}^{*}, \mu_{2}\right)
$$

for all mixed strategies $\mu_{1}$ and $\mu_{2}$ of Players 1 and 2, respectively. When these two inequalities hold up to an $\varepsilon>0$, the pair $\left(\mu_{1}^{*}, \mu_{2}^{*}\right)$ is called an $\varepsilon$-Nash equilibrium. 
The main problem we consider in our paper is the existence of Nash equilibria consisting of two-point strategies in game $\Gamma$. By definition, a two-point strategy is any pure strategy or mixed one with support consisting of at most two pure strategies, and will be denoted by $\alpha \delta_{x}+(1-\alpha) \delta_{y}$ with $0 \leq \alpha, x, y \leq 1$. Here and throughout the paper, $\delta_{t}$ is a degenerate probability distribution concentrated at point $t$ and will be identified with a pure strategy $t, 0 \leq t \leq 1$. So a two-point strategy $\alpha \delta_{x}+(1-\alpha) \delta_{y}$ prescribes a player to choose pure strategy $x$ with probability $\alpha$ and pure strategy $y$ with probability $1-\alpha$. It appears that two-point strategies (in spite of their simple form) play an essential role in describing Nash equilibria in wide classes of games.

To begin with, we recall three results concerning games $\Gamma$ on the unit square in which the conditions for the existence of Nash equilibria in two-point strategies are described. These results are a starting point for our study.

First background theorem belongs to Glicksberg (1952) and relates to the existence of Nash equilibrium in pure strategies for general $n$-person games. We quote his result only in the version for a two-person game on the unit square of the form (1), better suited for our discussion. We recall here that a real-valued function $f$ on $[0,1]$ is quasi-concave, when for each real $c$, the set $\{x: f(x) \geq c\}$ is convex. In the analogous way, a quasi-convex function is defined (after replacing " $\geq$ " by " $\leq$ )". Of course, every convex (concave) function is quasi-convex (quasi-concave).

Theorem A Assume that the payoff functions $F_{1}\left(x_{1}, x_{2}\right)$ and $F_{2}\left(x_{1}, x_{2}\right)$ are continuous on the unit square $[0,1]^{2}$, and quasi-concave in $x_{1}$ and $x_{2}$, respectively. Then game $\Gamma$ possesses a Nash equilibrium in pure strategies.

The second theorem belongs to Parthasarathy and Raghavan (1975) and can be seen as complementary to Theorem A.

Theorem $\mathbf{B}$ Assume that the payoff functions $F_{1}\left(x_{1}, x_{2}\right)$ and $F_{2}\left(x_{1}, x_{2}\right)$ are continuous on $[0,1]^{2}$, and $F_{1}\left(x_{1}, x_{2}\right)$ is concave in $x_{1}$. Then game $\Gamma$ possesses a Nash equilibrium of the form $\left(\delta_{a}, \beta \delta_{c}+(1-\beta) \delta_{d}\right)$ with $0 \leq \beta, a, c, d \leq 1$.

The third result (Theorem 2.3 in Radzik 1993), also essential for our further considerations, is a "convex version" of Theorem B.

Theorem $\mathbf{C}$ Assume that for $a=0,1$ the payoff functions $F_{1}\left(a, x_{2}\right)$ and $F_{2}\left(a, x_{2}\right)$ are continuous in $x_{2}$, and $F_{1}\left(x_{1}, x_{2}\right)$ is convex in $x_{1}$. Then game $\Gamma$ possesses a Nash equilibrium of the form $\left(\alpha \delta_{0}+(1-\alpha) \delta_{1}, \beta \delta_{c}+(1-\beta) \delta_{d}\right)$ with $0 \leq \alpha, \beta, c, d \leq 1$.

When the quasi-concavity of the payoff functions is omitted in the assumption of Theorem A, then there still exists a Nash equilibrium, but generally in mixed strategies. Glicksberg (1952) showed it for general $n$-person games. However we quote it only in the form analogous to that of Theorem A.

Theorem $\mathbf{D}$ Assume that the payoff functions $F_{1}\left(x_{1}, x_{2}\right)$ and $F_{2}\left(x_{1}, x_{2}\right)$ are continuous on the unit square $[0,1]^{2}$. Then game $\Gamma$ possesses a Nash equilibrium in mixed strategies.

Remark 1 It is known that the assumption about continuity of payoff functions in Theorem A cannot be weakened by upper semicontinuity (see Example 1 in Radzik 
and Ravindran 1989). However an open question is, if game $\Gamma$ with continuous payoff functions $F_{1}$ and $F_{2}$ and with function $F_{1}\left(x_{1}, x_{2}\right)$ quasi-concave in $x_{1}$ has a Nash equilibrium in two-point strategies. Note that Theorem B says that when quasi-concavity of both payoff functions in Theorem A is replaced by concavity of only one of them, game $\Gamma$ still has an "almost pure" Nash equilibrium. Further, Example 1 below shows that if we remove the continuity assumption for function $F_{2}$, both Theorems B and $\mathrm{C}$ are not longer true, and then game $\Gamma$ may have no Nash equilibrium at all. As far as the "concavity" assumption in Theorem B is concerned, Example 2 shows that it cannot be weakened by the assumption of quasi-concavity in $x_{1}$ for function $F_{1}\left(x_{1}, x_{2}\right)$. The answer is not known for the analogous question concerning Theorem $\mathrm{C}$.

Remark 2 It is worth mentioning that Theorems B and C have their generalizations (see Theorems 2.1 and 2.3 in Radzik (1993)). Namely, it may be seen as rather surprising that if in Theorem $\mathrm{B}$ the assumption about continuity of payoff functions $F_{1}$ and $F_{2}$ is replaced by boundedness on $[0,1]^{2}$, then game $\Gamma$ has an $\varepsilon$-Nash equilibria of the form $\left(\alpha \delta_{a}+(1-\alpha) \delta_{b}, \beta \delta_{c}+(1-\beta) \delta_{d}\right)$, for some $0 \leq \alpha, \beta, a, b, c, d \leq 1$ with $|a-b|<\varepsilon$.

Similarly, after removing "continuity" in Theorem $\mathrm{C}$, game $\Gamma$ also has an $\varepsilon$-Nash equilibrium of the form described there.

Example 1 Consider the game $\Gamma$ with the payoff functions $F_{1}$ and $F_{2}$ described by: $F_{1}\left(x_{1}, x_{2}\right)=0$ for $0 \leq x_{1}, x_{2} \leq 1$, and $F_{2}\left(x_{1}, x_{2}\right)=0$ for $0 \leq x_{1} \leq 1$ with $x_{2}=1$ and $F_{2}\left(x_{1}, x_{2}\right)=x_{2}$ otherwise. Since, as we easily see, each pure strategy $x_{2} \in[0,1]$ is strictly dominated by another one, there is no Nash equilibrium in this game. On the other hand, it is immediately seen that function $F_{1}\left(x_{1}, x_{2}\right)$ is both concave and convex in $x_{1}$, and function $F_{2}\left(x_{1}, x_{2}\right)$ is discontinuous. So, Theorems $\mathrm{B}$ and $\mathrm{C}$ are no longer true when the continuity assumptions are removed.

Example 2 Consider the game $\Gamma$ with the payoff functions described by the following:

$$
F_{1}\left(x_{1}, x_{2}\right)= \begin{cases}\left(1-2 x_{1}\right)\left(1-2 x_{2}\right) & \text { if } 0 \leq x_{1}, x_{2}<\frac{1}{2} \text { or } \frac{1}{2}<x_{1}, x_{2} \leq 1 \\ 0 & \text { otherwise }\end{cases}
$$

and

$$
F_{2}\left(x_{1}, x_{2}\right)=x_{2}^{2}-2 x_{1} x_{2} \quad \text { for } \quad 0 \leq x_{1}, x_{2} \leq 1
$$

One can easily verify that both payoff functions $F_{1}$ and $F_{2}$ are continuous on $[0,1]^{2}$, and function $F_{1}\left(x_{1}, x_{2}\right)$ is quasi-concave in $x_{1}$ (but not concave), and function $F_{2}\left(x_{1}, x_{2}\right)$ is convex in $x_{2}$. On the other hand, it is easy to show that game $\Gamma$ does not have a Nash equilibrium of the form $\eta^{*}=\left(\delta_{a}, \beta \delta_{c}+(1-\beta) \delta_{d}\right)$ with $c<d$ (the subcase $c=d$ is equivalent to $c<d$ with $\beta=0$ and thereby can be omitted). To prove it, suppose that game $\Gamma$ has a Nash equilibrium of the form $\eta^{*}$, and assume first that $0<c<1$ or $0<d<1$. Then $F_{2}\left(\eta^{*}\right)=\beta F_{2}(a, c)+(1-\beta) F_{2}(a, d)<$ $\max \left\{F_{2}(a, 0), F_{2}(a, 1)\right\}$, because function $F_{2}\left(x_{1}, x_{2}\right)$ is strictly convex in $x_{2}$. But this contradicts the fact that $\eta^{*}$ is a Nash equilibrium. Therefore $c=0$ and $d=1$, and let $\mu_{2}^{\beta}:=\beta \delta_{0}+(1-\beta) \delta_{1}$. 
Further, supposing that $a \neq \frac{1}{2}$ and $0<\beta<1$, we have: $F_{2}\left(a, \mu_{2}^{\beta}\right)=\beta F_{2}(a, 0)+$ $(1-\beta) F_{2}(a, 1)<\max \left\{F_{2}(a, 0), F_{2}(a, 1)\right\}$, because $F_{2}\left(x_{1}, 0\right) \neq F_{2}\left(x_{1}, 1\right)$ for $x_{1} \neq$ $\frac{1}{2}$. But this contradicts the optimality of strategy $\mu_{2}^{\beta}$ of Player 2 .

Similarly, the case $\left[a=\frac{1}{2}\right.$ and $\left.0 \leq \beta \leq 1\right]$ is impossible, because for $\beta>$ $0 F_{1}\left(\delta_{\frac{1}{2}}, \mu_{2}^{\beta}\right)=\beta F_{1}\left(\frac{1}{2}, 0\right)+(1-\beta) F_{1}\left(\frac{1}{2}, 1\right)=0<\frac{\beta}{2}=F_{1}\left(\frac{1}{4}, \mu_{2}^{\beta}\right)$, while for $\beta=0 F_{1}\left(\delta_{\frac{1}{2}}, \mu_{2}^{0}\right)=F_{1}\left(\frac{1}{2}, 1\right)=0<\frac{1}{2}=F_{1}\left(\frac{3}{4}, \mu_{2}^{0}\right)$, contradicting the optimality of strategy $\delta_{\frac{1}{2}}$ of Player 1 .

The impossibility of the two remaining cases, (I) $\left[a \neq \frac{1}{2}\right.$ and $\left.\beta=0\right]$, and (II) [ $a \neq \frac{1}{2}$ and $\beta=1$ ], can be shown similarly. Namely, in case (I), if $a<1$ then we have: $F_{1}\left(\delta_{a}, \mu_{2}^{0}\right)=F_{1}(a, 1) \leq \max \{0,(1-2 a) \cdot(-1)\}<1=F_{1}\left(1, \mu_{2}^{0}\right)$, which contradicts the optimality of strategy $\delta_{a}$ of Player 1 . On the other hand, if $a=1$ then $F_{2}\left(\delta_{1}, \mu_{2}^{0}\right)=F_{2}(1,1)=-1<0=F_{2}\left(\delta_{1}, 0\right)$, contradicting the optimality of strategy $\mu_{2}^{0}$ of Player 2 . The impossibility of the case (II) can be shown in almost exactly the same way as for (I), and is left to the reader.

\section{Definition of poorly convex functions}

In this section we introduce two basic notions of poorly convex/concave functions and pairwise poorly convex/concave families, together with a wide discussion. They play a fundamental role in the paper. In particular, they arise in the assumptions of our five main theorems presented in Sect. 4. The theory of poorly convex functions, needed for the proofs of the theorems, is developed in Sect. 5.

We begin with the following definition.

Definition 1 A function $f$ on $[a, b]$ is poorly convex (poorly concave) if for every $\left(x_{1}, x_{2}, \lambda\right)$ with $a \leq x_{1}<x_{2} \leq b$ and $0<\lambda<1$ there is a real number $p=$ $p\left(x_{1}, x_{2}, \lambda\right)$ with $0<p<1$ such that

$$
f\left(\lambda x_{1}+(1-\lambda) x_{2}\right) \leq(\geq) p\left(x_{1}, x_{2}, \lambda\right) f\left(x_{1}\right)+\left[1-p\left(x_{1}, x_{2}, \lambda\right)\right] f\left(x_{2}\right) .
$$

Remark 3 It is not difficult to see that the above definition can be straightforwardly generalized to poorly convex and poorly concave functions defined on convex subsets $U$ of an arbitrary vector space. It suffices only to replace the set of vectors $\left\{\left(x_{1}, x_{2}, \lambda\right)\right.$ : $\left.a \leq x_{1}<x_{2} \leq b, 0<\lambda<1\right\}$ by the set $\left\{\left(x_{1}, x_{2}, \lambda\right): x_{1}, x_{2} \in U, x_{1} \neq x_{2}, 0<\right.$ $\lambda<1\}$. However, in this paper we restrict our attention only to such functions on an interval of the real line which are needed to formulate our main theorems in Sect. 4.

Now we give a proposition which presents an equivalent but a little simpler characterization of poorly convex and poorly concave functions, more convenient for our further considerations. To formulate it, for brevity, we introduce the notation

$$
\bar{u}:=\left(u_{1}, u_{2}, u_{3}\right),
$$

and, for a given interval $[a, b]$, we define the set 


$$
Q_{[a, b]}:=\left\{\bar{u}: a \leq u_{1}<u_{2}<u_{3} \leq b\right\}
$$

Proposition 1 A function $f$ is poorly convex (poorly concave) on $[a, b]$ if and only if there is a positive function $T$ defined on the set $Q_{[a, b]}$, such that for all $\bar{u} \in Q_{[a, b]}$, the following inequality hold:

$$
f\left(u_{2}\right)-f\left(u_{1}\right) \leq(\geq) T(\bar{u})\left[f\left(u_{3}\right)-f\left(u_{2}\right)\right] .
$$

Proof $(\Rightarrow)$ Fix arbitrarily $\left(x_{1}, x_{2}, \lambda\right)$ with $a \leq x_{1}<x_{2} \leq b$ and $0<\lambda<1$. Let $u_{1}=x_{1}, u_{3}=x_{2}$ and $u_{2}=\lambda x_{1}+(1-\lambda) x_{2}$, whence $\lambda=\frac{u_{3}-u_{2}}{u_{3}-u_{1}}$. So we can write (2) as dependent only on $\left(u_{1}, u_{2}, u_{3}\right)$, in the form equivalent to (4) with

$$
T(\bar{u})=\frac{1-p\left(u_{1}, u_{3}, \frac{u_{3}-u_{2}}{u_{3}-u_{1}}\right)}{p\left(u_{1}, u_{3}, \frac{u_{3}-u_{2}}{u_{3}-u_{1}}\right)},
$$

as it is easy to verify. This equality well defines a positive function $T$ on $Q_{[a, b]}$ because $\bar{u} \in Q_{[a, b]}$, whence $a \leq u_{1}<u_{3} \leq b$ and $0<\frac{u_{3}-u_{2}}{u_{3}-u_{1}}<1$.

$(\Leftarrow)$ Fix arbitrarily $\bar{u} \in Q_{[a, b]}$. Obviously, for some $0<\lambda<1, u_{2}=\lambda u_{1}+(1-$ $\lambda) u_{3}$. Putting now $u_{1}=x_{1}, u_{3}=x_{2}$ and $u_{2}=\lambda x_{1}+(1-\lambda) x_{2}$ in (4), we easily check that this inequality is equivalent to (2) with

$$
p\left(x_{1}, x_{2}, \lambda\right)=\frac{1}{1+T\left(x_{1}, \lambda x_{1}+(1-\lambda) x_{2}, x_{2}\right)} .
$$

The value $p\left(x_{1}, x_{2}, \lambda\right)$ is well defined, because $a \leq x_{1}<x_{2} \leq b, 0<\lambda<1$ and $\left(x_{1}, \lambda x_{1}+(1-\lambda) x_{2}, x_{2}\right) \in Q_{[a, b]}$. This ends the proof.

Proposition 1 allows us to give an equivalent definition of poorly convex and poorly concave functions on an interval $[a, b]$, which is better suited for our further considerations.

Definition 2 A function $f$ on $[a, b]$ is poorly convex (poorly concave) if there is a positive function $T$ defined on the set $Q_{[a, b]}$ such that for all $\bar{u} \in Q_{[a, b]}$, inequality (4) holds. Then function $f$ is also called $T$-convex ( $T$-concave) on $[a, b]$.

Now we are ready to give our second basic definition.

Definition 3 Let $\mathcal{X}$ be an index set. A family $\left\{f_{\alpha}: \alpha \in \mathcal{X}\right\}$ of functions on interval $[a, b]$ is pairwise poorly convex (pairwise poorly concave) if for every pair $\alpha, \beta \in \mathcal{X}$ there is a positive function $T$ on $Q_{[a, b]}$ such that the functions $f_{\alpha}$ and $f_{\beta}$ are $T$-convex ( $T$-concave) on $[a, b]$.

Remark 4 One can easily see that function $T$ related to a poorly convex function (in Definition 1) neither has to be continuous nor unique. A wide analysis of poorly convex functions and pairwise poorly convex families of functions is presented in Sects. 5 and 6. In particular, in Proposition 3 we give necessary and sufficient conditions 
for a continuous function to be poorly convex, which shows that the family of poor convex (concave) functions on an interval is much richer than that of convex (concave) ones. We also mention here the relations between convex and poorly convex functions. Namely, every convex (concave) function $f$ on an interval $[a, b]$ is $T$-convex (T-concave) on $[a, b]$ with function $T$ of the form $T(\bar{u})=\frac{u_{2}-u_{1}}{u_{3}-u_{2}}$. Therefore every family of convex (concave) functions is a pairwise poorly convex (concave) one, but not conversely. The pairwise poorly convex (concave) families are substantially richer and play a fundamental role in our considerations. At the end of Sect. 6 we give two examples (Examples 3 and 4) of pairwise poorly convex families consisting of non-convex standard functions.

Remark 5 An easy analysis of inequality (4) shows that poor convexity of a function $f$ on an interval $[a, b]$ can also be defined by requiring for all $a \leq u_{1}<u_{2}<$ $u_{3} \leq b$ the following two implications: $\left[f\left(u_{2}\right)=f\left(u_{1}\right)\right] \Rightarrow\left[f\left(u_{3}\right) \geq f\left(u_{2}\right)\right]$, and $\left[f\left(u_{2}\right)>f\left(u_{1}\right)\right] \Rightarrow\left[f\left(u_{3}\right)>f\left(u_{2}\right)\right]$. This latter definition shows that poor convexity is indeed stronger than quasi-convexity. However, poor convexity can be also seen as a very minor strengthening of quasi-convexity, since every continuous quasi-convex function on an interval can be approximated by a sequence of continuous poorly convex functions in the topology of uniform convergence (see Proposition 4 in Sect. 5). Similar remarks can be made for poor concavity. ${ }^{1}$

\section{Main theorems}

In this section we discuss possible generalizations of Theorems B and C from Sect. 2. As a result, five new Theorems 1-5 are proposed (their proofs will be given in Sect. 7). The basic question is how far we can weaken the assumptions of Theorems B and $\mathrm{C}$, still having (in game $\Gamma$ ) the existence of a Nash equilibrium of the form described there. Example 2 given in Sect. 2 shows that Theorem B is false when we replace "concave" by "quasi-concave" in its assumptions. Hence a very intriguing question is whether Theorem B will remain true if we replace concavity assumption by "something between" concavity and quasi-concavity. The same question is essential for Theorem C. Just these two questions are basic for our study and we will show that the answer for both of them is positive.

Below we formulate the main five results of the paper. Their proofs are given in Sect. 7 which is preceded by the two auxiliary Sects. 5 and 6, where a theory of poorly convex functions is developed. The first two theorems generalize Theorems B and C.

Theorem 1 Assume that the payoff functions $F_{1}\left(x_{1}, x_{2}\right)$ and $F_{2}\left(x_{1}, x_{2}\right)$ are continuous on the unit square $[0,1]^{2}$, and $\left\{F_{1}\left(\cdot, x_{2}\right): x_{2} \in[0,1]\right\}$ is a pairwise poorly concave family of functions on $[0,1]$. Then game $\Gamma$ possesses a Nash equilibrium of the form $\left(\delta_{a}, \beta \delta_{c}+(1-\beta) \delta_{d}\right)$, for some $0 \leq \beta, a, c, d \leq 1$.

Theorem 2 Assume for $a=0,1$ that the payoff functions $F_{1}(a, \cdot)$ and $F_{2}(a, \cdot)$ are continuous, and $\left\{F_{1}\left(\cdot, x_{2}\right): x_{2} \in[0,1]\right\}$ is a pairwise poorly convex family of

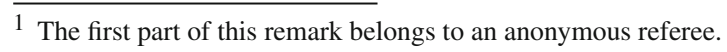


functions on $[0,1]$. Then game $\Gamma$ possesses a Nash equilibrium of the form $\left(\alpha \delta_{0}+\right.$ $\left.(1-\alpha) \delta_{1}, \beta \delta_{c}+(1-\beta) \delta_{d}\right)$, for some $0 \leq \alpha, \beta, c, d \leq 1$.

The next two results can be seen as a completion of Theorem 1. They show that players" "simple strategies" (with two-point supports) arising in Theorems 1 and 2 are quite satisfactory for them in many game cases.

Theorem 3 Assume that $F_{2}\left(x_{1}, x_{2}\right)$ is continuous on the unit square $[0,1]^{2}$, and $\left\{F_{1}\left(\cdot, x_{2}\right): x_{2} \in[0,1]\right\}$ is a pairwise poorly concave family of continuous functions on $[0,1]$. If game $\Gamma$ possesses a Nash equilibrium $(\mu, v)$ with a payoff vector $\left(f_{1}, f_{2}\right)$ then it also has a Nash equilibrium of the form $\left(\mu, \beta \delta_{c}+(1-\beta) \delta_{d}\right)$ for some $0 \leq$ $\beta, c, d \leq 1$, with a payoff vector $\left(f_{1}^{\prime}, f_{2}^{\prime}\right)$ having the same second component $f_{2}^{\prime}=f_{2}$.

Remark 6 We do not know if the assumptions of Theorem 3 guarantee the existence of a Nash equilibrium in game $\Gamma$. However, it can facilitate solving this problem in concrete cases of $\Gamma$.

Theorem 3 leads immediately to the next one.

Theorem 4 Assume that the payoff functions $F_{1}\left(x_{1}, x_{2}\right)$ and $F_{2}\left(x_{1}, x_{2}\right)$ are continuous on the unit square $[0,1]^{2}$, and $\left\{F_{1}\left(\cdot, x_{2}\right): x_{2} \in[0,1]\right\}$ and $\left\{F_{2}\left(x_{1}, \cdot\right)\right.$ : $\left.x_{1} \in[0,1]\right\}$ are pairwise poorly concave families of functions on $[0,1]$. Then for any Nash equilibrium in game $\Gamma$ there is also a Nash equilibrium of the form $\left(\alpha \delta_{a}+(1-\alpha) \delta_{b}, \beta \delta_{c}+(1-\beta) \delta_{d}\right)$ for some $0 \leq \alpha, \beta, a, b, c, d \leq 1$, with the same payoff vector.

Remark 7 Theorem D from Sect. 2 implies that under the assumption of Theorem 4 , game $\Gamma$ always has a Nash equilibrium in mixed strategies. Hence, the result of Theorem 4 can be interpreted that any Nash equilibrium in mixed strategies has an "equivalent" Nash equilibrium in two-point strategies in this game.

The last theorem is a "discontinuous modification" of Theorem 2.

Theorem 5 Assume that $\left\{F_{1}\left(\cdot, x_{2}\right): x_{2} \in[0,1]\right\}$ and $\left\{F_{2}\left(x_{1}, \cdot\right): x_{1} \in[0,1]\right\}$ are pairwise poorly convex families of functions on $[0,1]$. Then game $\Gamma$ has a Nash equilibrium of the form $\left(\alpha \delta_{0}+(1-\alpha) \delta_{1}, \beta \delta_{0}+(1-\beta) \delta_{1}\right)$ with $0 \leq \alpha, \beta \leq 1$.

Remark 8 In general, Theorems 1 and 2 are no longer true when we admit for payoff functions $F_{1}$ and $F_{2}$ to be discontinuous (the same was shown for Theorems $\mathrm{B}$ and $\mathrm{C}$ in Example 1 in Sect. 2). Moreover, it may happen then that there are no Nash equilibria at all. However the author does not know whether after removing the continuity assumption in Theorems 1 and 2, the games still have $\varepsilon$-Nash equilibria for all $\varepsilon>0$ [we recall that this is true for Theorems B and C (see Remark 2)]. The next question is whether Theorems 3 and 4 still remain true after changing "poorly concave" with "poorly convex".

Remark 9 Some "discrete" versions of poorly concave games considered in this paper have been also studied in the literature, where the spaces of players' pure strategies are finite, and payoff functions are assumed to satisfy a discrete version of the pairwise 
poor concavity property. In paper Radzik (2000) some counterparts of Theorems 1-4 are presented for matrix games, while in Połowczuk (2006) for bimatrix games. A discrete versions of these theorems for poorly concave $n$-person games can be found in Połowczuk et al. (2007).

\section{Theory of poorly convex functions}

Pairwise poor convexity and poor concavity are basic properties of families of functions considered in the assumptions of our main Theorems 1-5. Having this in mind, we devote this section to develop a basic theory of such functions. Among other things, we find necessary and sufficient conditions for families of continuous functions to be pairwise poorly convex. This gives us tools for constructing pairwise poorly convex families and thereby to show that the class of such families is substantially richer than the family of convex functions (Examples 3 and 4 in Sect. 6). We mainly study poorly convex functions, because the results obtained can be trivially modified to analogous ones for poorly concave functions. We do it in Propositions 2-7.

In the first proposition, functions $f$ and $g$ are considered to be defined on an interval $[a, b]$. We list here five basic properties of poorly convex functions. Obviously, the proposition remains true after replacing "convex" by "concave" in it.

Proposition 2 The following statements hold:

(a) a function $f$ is poorly convex if and only if $-f$ is poorly concave;

(b) every convex function $f$ is poorly convex;

(c) every poorly convex function $f$ is quasi-convex;

(d) if $f$ is $T_{1}$ - and $T_{2}$-convex then $f$ is $\left(\alpha T_{1}+\beta T_{2}\right)$-convex for all $\alpha, \beta \geq 0$ with $\alpha+\beta=1$;

(e) if $f$ and $g$ are $T$-convex functions then for every $\alpha, \beta \geq 0$ the function $\alpha f+\beta g$ is also $T$-convex.

Proof Statements (a), (d) and (e) are simple consequences of Definition 1. Statement (b) was justified in Remark 4. Therefore, it suffices to show statement (c).

By assumption, function $f$ satisfies inequality (4). Let $c \in R, a \leq u_{1}<u_{2}<$ $u_{3} \leq b$, and assume that $f\left(u_{1}\right) \leq c$ and $f\left(u_{3}\right) \leq c$. Hence, we can conclude with the help of inequality (4) as follows:

$$
f\left(u_{2}\right)(1+T(\bar{u})) \leq f\left(u_{1}\right)+T(\bar{u}) f\left(u_{3}\right) \leq c+c T(\bar{u})=c(1+T(\bar{u})),
$$

and thereby $f\left(u_{2}\right) \leq c$, because $T(\bar{u})>0$. Therefore the set $\{u: f(u) \leq c\}$ is convex which, in view of arbitrarity of $c$, implies that function $f$ is quasi-convex.

The next proposition characterizes an arbitrary poorly convex continuous function. To express it, we need to define the following two quantities for a function $f$ on an interval $[a, b]$ :

$$
\tau_{f}:=\sup \{x \in[a, b]: f \text { is strictly decreasing on }[a, x]\}
$$

and 


$$
\tau^{f}:=\inf \{x \in[a, b]: f \text { is strictly increasing on }[x, b]\} .
$$

Obviously, $\tau_{f} \leq \tau^{f}$. Both these quantities are basic for our subsequent considerations.

Proposition 3 A continuous function $f$ on $[a, b]$ is poorly convex if and only if there are constants $c \leq d$ in $[a, b]$ such that $f$ is strictly decreasing on $[a, c]$, strictly increasing on $[d, b]$, and constant on $[c, d]$. Then $c=\tau_{f}$ and $d=\tau^{f}$.

Proof $(\Rightarrow)$ Since this part of the proposition is obvious in the case $\tau_{f}=\tau^{f}$, we can assume $\tau_{f}<\tau^{f}$. Suppose first that function $f$ is not constant on $\left[\tau_{f}, \tau^{f}\right]$. Hence there are $u_{1}<u_{2}$ in the interval $\left(\tau_{f}, \tau^{f}\right)$ such that $f\left(u_{1}\right)>f\left(u_{2}\right)$ or $f\left(u_{1}\right)<f\left(u_{2}\right)$. We consider these two cases.

Case 1: $f\left(u_{1}\right)>f\left(u_{2}\right)$.

By Definition 2, function $f$ is $T$-convex for some positive function $T$ defined on the set $Q_{[a, b]}$. Let $a \leq u^{\prime}<u^{\prime \prime}<u_{1}<u_{2} \leq b$ and denote $T_{1}:=T\left(u^{\prime}, u^{\prime \prime}, u_{1}\right)$ and $T_{2}:=T\left(u^{\prime \prime}, u_{1}, u_{2}\right)$. Then (4) implies that

$$
f\left(u^{\prime \prime}\right)-f\left(u^{\prime}\right) \leq T_{1}\left[f\left(u_{1}\right)-f\left(u^{\prime \prime}\right)\right] \leq T_{1} T_{2}\left[f\left(u_{2}\right)-f\left(u_{1}\right)\right],
$$

whence we easily deduce that $f\left(u^{\prime}\right)>f\left(u^{\prime \prime}\right)$ and $f\left(u^{\prime \prime}\right)>f\left(u_{1}\right)$, because of $f\left(u_{1}\right)>$ $f\left(u_{2}\right)$ and $T_{1}, T_{2}>0$. But this, in view of the arbitrarity of $u^{\prime}, u^{\prime \prime}$, proves that function $f$ is strictly decreasing in the interval $\left[a, u_{1}\right]$. However this contradicts (7) because of $u_{1} \in\left(\tau_{f}, \tau^{f}\right)$. Therefore Case 1 cannot hold.

Case 2: $f\left(u_{1}\right)<f\left(u_{2}\right)$.

This case is also impossible. To show it, it suffices to repeat the reasoning of Case 1 , replacing parameters $u^{\prime}, u^{\prime \prime}, u_{1}$ and $u_{2}$ by $u_{1}, u_{2}, u^{\prime}$ and $u^{\prime \prime}$, respectively. The clear details are omitted.

Therefore $f\left(u_{1}\right)=f\left(u_{2}\right)$. But this, in view of the arbitrarity of $u_{1}, u_{2}$, proves that function $f$ is constant in the interval $\left[\tau_{f}, \tau^{f}\right]$. This completes the proof of part $(\Rightarrow)$.

$(\Leftarrow)$ We can directly verify that function $f$ satisfies $(4)$ for positive function $T$ defined on $Q_{[a, b]}$ in the following way:

$$
T(\bar{u}):=\left\{\begin{array}{ccc}
\frac{f\left(u_{2}\right)-f\left(u_{1}\right)}{f\left(u_{3}\right)-f\left(u_{2}\right)} & \text { if } & {\left[f\left(u_{2}\right)-f\left(u_{1}\right)\right]\left[f\left(u_{3}\right)-f\left(u_{2}\right)\right]>0} \\
1 & \text { otherwise. }
\end{array}\right.
$$

This completes the proof of Proposition 3.

Now we give a proposition showing that poor convexity is a very minor strengthening of quasi-convexity.

Proposition 4 Any continuous quasi-convex function $f$ on $[a, b]$ can be approximated by a sequence $\left(f_{n}\right)$ of poorly convex continuous functions in the topology of uniform convergence.

Proof For $n=1,2, \ldots$, let us choose real numbers $\Delta_{n}$ to satisfy: $\left|f\left(x^{\prime}\right)-f\left(x^{\prime \prime}\right)\right| \leq$ $\Delta_{n}$ if $\left|x^{\prime}-x^{\prime \prime}\right| \leq \frac{1}{n}$ in $[a, b]$. Since every continuous function on an interval is also uniformly continuous, we may choose $\Delta_{n} \rightarrow 0$ as $n \rightarrow \infty$. 
Let us fix $c$ satisfying $f(c)=\min _{u \in[a, b]} f(u)$, and firstly assume that $a<c<b$. For a natural $n$, we define two sequences $\left(x_{0}, x_{1}, \ldots, x_{n}\right)$ and $\left(y_{0}, y_{1}, \ldots, y_{n}\right)$ by the following:

$$
x_{k}=a+k \frac{c-a}{n} \quad \text { and } \quad y_{k}=c-k \frac{b-c}{n}, \quad k=0,1, \ldots, n .
$$

Note that $a=x_{0}<x_{1}<\ldots<x_{n}=c=y_{n}<y_{n-1}<\ldots<y_{0}=b$. Now, let $f_{n}$ be a function on $[a, b]$ determined by the following two conditions:

(i) $f_{n}\left(x_{k}\right)=f_{n}\left(y_{k}\right)=f\left(x_{k}\right)-\sum_{i=0}^{k} \frac{1}{n^{i+1}}$ for $k=0,1, \ldots, n$, and

(ii) function $f_{n}$ is linear on all the intervals of the form $\left[x_{k}, x_{k+1}\right]$ and $\left[y_{k+1}, y_{k}\right], k=$ $0,1, \ldots, n-1$.

By Proposition 3, function $f$ is nonincreasing on $[a, c]$ and nondecreasing on $[c, b]$. Hence, one can easily conclude with the help of conditions (i) and (ii) that function $f_{n}$ is continuous on $[a, b]$, strictly decreasing on $[a, c]$ and strictly increasing on $[c, b]$. Therefore, Proposition 3 can be used again to conclude that function $f_{n}$ is poorly convex on $[a, b]$. Besides we easily deduce that

$$
\max _{u \in[a, b]}\left|f(u)-f_{n}(u)\right| \leq \Delta_{n}+\sum_{i=0}^{n} \frac{1}{n^{i+1}}<\Delta_{n}+\sum_{i=0}^{\infty} \frac{1}{n^{i+1}}=\Delta_{n}+\frac{1}{n-1}
$$

which immediately completes the proof in the case $a<c<b$. When $a=c$ or $b=c$, the reasoning can be repeated with only the sequence $\left\{y_{k}\right\}$ or $\left\{x_{k}\right\}$, respectively.

In the next three propositions we discuss the question of the conditions guaranteeing for a pair of continuous poorly convex functions to be pairwise poorly convex. However, for their proofs, we need the following lemma.

Lemma 1 Let $f$ and $g$ be poorly convex functions on $[a, b]$. Then the pair $\{f, g\}$ is pairwise poorly convex if and only if for every $\bar{u} \in Q_{[a, b]}$ satisfying

$$
f\left(u_{1}\right)<f\left(u_{2}\right)<f\left(u_{3}\right) \text { and } g\left(u_{1}\right)>g\left(u_{2}\right)>g\left(u_{3}\right),
$$

or

$$
f\left(u_{1}\right)>f\left(u_{2}\right)>f\left(u_{3}\right) \text { and } g\left(u_{1}\right)<g\left(u_{2}\right)<g\left(u_{3}\right),
$$

there is an $h>0$ such that

$$
f\left(u_{2}\right)-f\left(u_{1}\right) \leq h\left[f\left(u_{3}\right)-f\left(u_{2}\right)\right]
$$

and

$$
g\left(u_{2}\right)-g\left(u_{1}\right) \leq h\left[g\left(u_{3}\right)-g\left(u_{2}\right)\right] .
$$


Proof By Definition 2, there are some positive functions $T_{1}(\bar{u})$ and $T_{2}(\bar{u})$ on $Q_{[a, b]}$ such that function $f$ is $T_{1}$-convex and function $g$ is $T_{2}$-convex on $[a, b]$.

$(\Rightarrow)$ In view of Definitions 2 and 3, this part of the lemma is obvious.

$(\Leftarrow)$ Let us arbitrarily fix $\bar{u} \in Q_{[a, b]}$. If (9) or (10) are satisfied, pairwise poor convexity of the pair $\{f, g\}$ follows by the assumption. Therefore, for the rest of our considerations, we can assume that (9) and (10) do not hold. To complete the proof of the lemma, it suffices to show that there is a number $h>0$ for which (11) and (12) hold. We consider several cases.

Case 1: $f\left(u_{1}\right) \leq f\left(u_{2}\right)$ and $g\left(u_{1}\right) \leq g\left(u_{2}\right)$.

Hence, with the help of (4), we can conclude as follows:

$$
f\left(u_{3}\right)-f\left(u_{2}\right) \geq \frac{1}{T_{1}(\bar{u})}\left[f\left(u_{2}\right)-f\left(u_{1}\right)\right] \geq \frac{1}{\max \left\{T_{1}(\bar{u}), T_{2}(\bar{u})\right\}}\left[f\left(u_{2}\right)-f\left(u_{1}\right)\right],
$$

and thereby (11) follows for $h=\frac{1}{\max \left\{T_{1}(\bar{u}), T_{2}(\bar{u})\right\}}$. Exactly in the same way, replacing $f$ and $T_{1}$ by $g$ and $T_{2}$, respectively, we show (12) for the same $h$.

Case 2: $f\left(u_{1}\right) \geq f\left(u_{2}\right)$ and $g\left(u_{1}\right) \geq g\left(u_{2}\right)$.

Now we can repeat the reasoning of Case 1 to get the validity of (11) and (12) for $h=\frac{1}{\min \left\{T_{1}(\bar{u}), T_{2}(\bar{u})\right\}}$.

Case 3: $f\left(u_{2}\right) \leq f\left(u_{3}\right)$ and $g\left(u_{2}\right) \leq g\left(u_{3}\right)$.

Then we have:

$$
f\left(u_{2}\right)-f\left(u_{1}\right) \leq T_{1}(\bar{u})\left[f\left(u_{3}\right)-f\left(u_{2}\right)\right] \leq \max \left\{T_{1}(\bar{u}), T_{2}(\bar{u})\right\}\left[f\left(u_{3}\right)-f\left(u_{2}\right)\right]
$$

and

$$
g\left(u_{2}\right)-g\left(u_{1}\right) \leq T_{2}(\bar{u})\left[g\left(u_{3}\right)-g\left(u_{2}\right)\right] \leq \max \left\{T_{1}(\bar{u}), T_{2}(\bar{u})\right\}\left[g\left(u_{3}\right)-g\left(u_{2}\right)\right]
$$

Consequently, (11) and (12) hold for $h=\max \left\{T_{1}(\bar{u}), T_{2}(\bar{u})\right\}$.

Case 4: $f\left(u_{2}\right) \geq f\left(u_{3}\right)$ and $g\left(u_{2}\right) \geq g\left(u_{3}\right)$.

Now we can repeat the reasoning of Case 3 to get the validity of (11) and (12) for $h=\min \left\{T_{1}(\bar{u}), T_{2}(\bar{u})\right\}$.

Case 5: $f\left(u_{1}\right) \geq f\left(u_{2}\right) \leq f\left(u_{3}\right)$.

Then (11) and (12) hold for $h=T_{2}(\bar{u})$.

Case 6: $g\left(u_{1}\right) \geq g\left(u_{2}\right) \leq g\left(u_{3}\right)$.

Then (11) and (12) hold for $h=T_{1}(\bar{u})$.

One can easily deduce that if (9) and (10) do not hold, then one of cases 1-6 must hold. This ends the proof of the lemma.

Proposition 5 Let $f$ and $g$ be continuous and poorly convex functions on $[a, b]$, satisfying $\left[\tau_{f}, \tau^{f}\right] \cap\left[\tau_{g}, \tau^{g}\right] \neq \varnothing$. Then the pair $\{f, g\}$ is pairwise poorly convex on $[a, b]$.

Proof Let $\bar{u} \in Q_{[a, b]}$ and suppose that (9) holds. Then Proposition 3 applied to functions $f$ and $g$ implies that $u_{2}>\tau^{f}$ and $u_{2}<\tau_{g}$, whence $\tau^{f}<\tau_{g}$. But this contradicts the assumption $\left[\tau_{f}, \tau^{f}\right] \cap\left[\tau_{g}, \tau^{g}\right] \neq \emptyset$. 
On the other hand, when we suppose that (10) holds, in a similar way we get $\tau^{g}<\tau_{f}$, contradicting the assumption again. Therefore, (9) and (10) do not hold for $\bar{u} \in Q_{[a, b]}$, which by Lemma 1 ends the proof.

The next proposition considers the second case $\left[\tau_{f}, \tau^{f}\right] \cap\left[\tau_{g}, \tau^{g}\right]=\emptyset$. To formulate it, we need to introduce the following notation:

$$
Q_{g}^{f}:=\left\{\bar{u}: \tau^{f} \leq u_{1}<u_{2}<u_{3} \leq \tau_{g}\right\}
$$

Proposition 6 Let $f$ and $g$ be continuous and poorly convex functions on $[a, b]$ with $\tau^{f}<\tau_{g}$. Then the pair $\{f, g\}$ is pairwise poorly convex on $[a, b]$ if and only if

$$
\frac{f\left(u_{2}\right)-f\left(u_{1}\right)}{f\left(u_{3}\right)-f\left(u_{2}\right)} \leq \frac{g\left(u_{2}\right)-g\left(u_{1}\right)}{g\left(u_{3}\right)-g\left(u_{2}\right)} \quad \text { for } \bar{u} \in Q_{g}^{f} .
$$

Proof In view of $\tau^{f}<\tau_{g}$, Proposition 3 implies that function $f$ is strictly increasing in $\left[\tau^{f}, \tau_{g}\right]$, and function $g$ is strictly decreasing in $\left[\tau^{f}, \tau_{g}\right]$. Hence, it follows that

$$
f\left(u_{1}\right)<f\left(u_{2}\right)<f\left(u_{3}\right) \text { and } \quad g\left(u_{1}\right)>g\left(u_{2}\right)>g\left(u_{3}\right) \quad \text { for } \bar{u} \in Q_{g}^{f} .
$$

$(\Rightarrow)$ By Definitions 2 and 3, it follows that there is a positive function $T$ on $Q_{[a, b]}$ such that for $\bar{u} \in Q_{[a, b]}$

$$
f\left(u_{2}\right)-f\left(u_{1}\right) \leq T(\bar{u})\left[f\left(u_{3}\right)-f\left(u_{2}\right)\right]
$$

and

$$
g\left(u_{2}\right)-g\left(u_{1}\right) \leq T(\bar{u})\left[g\left(u_{3}\right)-g\left(u_{2}\right)\right]
$$

which, by (14), immediately implies (13).

$(\Leftarrow)$ Assume now that $(13)$ holds. To end the proof it suffices to show that there is a positive function $T$ defined on the set $Q_{[a, b]}$ such that inequalities (15) and 16) hold for all $\bar{u} \in Q_{[a, b]}$.

Case 1: $\bar{u} \in Q_{g}^{f}$.

Let us define $T(\bar{u})$ as any positive value satisfying the inequalities,

$$
\frac{f\left(u_{2}\right)-f\left(u_{1}\right)}{f\left(u_{3}\right)-f\left(u_{2}\right)} \leq T(\bar{u}) \leq \frac{g\left(u_{2}\right)-f\left(u_{1}\right)}{g\left(u_{3}\right)-g\left(u_{2}\right)},
$$

which is possible because of (13). But this together with (14) imply (15) and (16).

Case 2: $\bar{u} \notin Q_{g}^{f}, f\left(u_{1}\right)<f\left(u_{2}\right)<f\left(u_{3}\right)$ and $g\left(u_{1}\right)>g\left(u_{2}\right)>g\left(u_{3}\right)$.

One can easily see with the help of Proposition 3 that

$$
\tau^{f}<u_{2}<\tau_{g}
$$

However, this implies that 


$$
u_{1}<\tau^{f} \text { or } u_{3}>\tau_{g}
$$

since otherwise, it would contradict $\bar{u} \notin Q_{g}^{f}$.

Consider now the first subcase

$$
\text { (A ) : } u_{1} \leq \tau^{f} \quad \text { and } \quad u_{3} \geq \tau_{g}
$$

where one of the inequalities is strict. By Proposition 3 and the assumption $\tau^{f}<\tau_{g}$, function $f$ is nonincreasing on $\left[u_{1}, \tau^{f}\right]$ and strictly increasing on $\left[\tau_{g}, u_{3}\right]$, and function $g$ is strictly decreasing on $\left[u_{1}, \tau^{f}\right]$ and nondecreasing on $\left[\tau_{g}, u_{3}\right]$. It implies that for $u_{1}^{\prime}=\tau^{f}$ and $u_{3}^{\prime}=\tau_{g}$ we have

$$
\begin{array}{ll}
f\left(u_{2}\right)-f\left(u_{1}\right) \leq f\left(u_{2}\right)-f\left(u_{1}^{\prime}\right) \quad \text { and } \quad g\left(u_{2}\right)-g\left(u_{1}\right) \leq g\left(u_{2}\right)-g\left(u_{1}^{\prime}\right), \\
f\left(u_{3}\right)-f\left(u_{2}\right) \geq f\left(u_{3}^{\prime}\right)-f\left(u_{2}\right) \quad \text { and } \quad g\left(u_{3}\right)-g\left(u_{2}\right) \geq g\left(u_{3}^{\prime}\right)-g\left(u_{2}\right),
\end{array}
$$

and $u_{1}^{\prime}<u_{2}<u_{3}^{\prime}$, because of (18) and (19).

Therefore the vector $\bar{u}^{*}=\left(u_{1}^{\prime}, u_{2}, u_{3}^{\prime}\right)$ satisfies $\bar{u}^{*} \in Q_{g}^{f}$ and consequently, by Case 1 , for some $T\left(\bar{u}^{*}\right)>0$,

$$
f\left(u_{2}\right)-f\left(u_{1}^{\prime}\right) \leq T\left(\bar{u}^{*}\right)\left[f\left(u_{3}^{\prime}\right)-f\left(u_{2}\right)\right]
$$

and

$$
g\left(u_{2}\right)-g\left(u_{1}^{\prime}\right) \leq T\left(\overline{u^{*}}\right)\left[g\left(u_{3}^{\prime}\right)-g\left(u_{2}\right)\right] .
$$

But this together with (20) and (21) imply that inequalities (15) and (16) hold for $T(\bar{u})=T\left(\bar{u}^{*}\right)$ in the first subcase (19) considered.

The two remaining subcases can be described by the conditions, (B): $u_{1} \leq \tau^{f}<$ $u_{2}<u_{3}<\tau_{g}$, and $(\mathrm{C}): \tau^{f}<u_{1}<u_{2}<\tau_{g}<u_{3}$. We can analyze them, by the exact repetition of reasoning in subcase (A), changing only $u_{3}^{\prime}$ with $u_{3}$ for subcase (B), and $u_{1}^{\prime}$ with $u_{1}$ for subcase $(\mathrm{C})$. In both subcases, the results obtained will be the same as in subcase (A). The clear details are omitted.

Case 3: $\bar{u} \notin Q_{g}^{f}, f\left(u_{1}\right)>f\left(u_{2}\right)>f\left(u_{3}\right)$ and $g\left(u_{1}\right)<g\left(u_{2}\right)<g\left(u_{3}\right)$.

This case cannot occur. Namely, then Proposition 3 would imply $u_{1}<u_{2} \leq \tau^{f}$ and $\tau_{g} \leq u_{2}<u_{3}$ which is impossible, because of the assumption $\tau^{f}<\tau_{g}$.

Now, taking into account Lemma 1 and conditions describing Cases $1-3$, we easily deduce that the pair of functions $\{f, g\}$ is pairwise poorly convex on $[a, b]$, completing the proof of the proposition.

One can see the verification of condition (13) as somewhat complex. The next proposition is a special version of the previous one where verification of (13) is replaced by a much easiercondition. 
Proposition 7 Let $f$ and $g$ be continuous and poorly convex functions on $[a, b]$ with $\tau^{f}<\tau_{g}$. Assume that there are continuous derivatives $f^{\prime}$ and $g^{\prime}$ on $\left(\tau^{f}, \tau_{g}\right)$, with $g^{\prime} \neq 0$. Then the pair of functions $\{f, g\}$ is pairwise poorly convex on $[a, b]$ if and only if the function $G(u)=f^{\prime}(u) / g^{\prime}(u)$ is nonincreasing on $\left(\tau^{f}, \tau_{g}\right)$.

Proof $(\Rightarrow)$ Let $\tau^{f}<u_{1}<u_{2}<u_{3}<u_{4}<\tau_{g}$. By Proposition 3, functions $f$ and $g$ are strictly increasing and strictly decreasing in interval $\left[\tau^{f}, \tau_{g}\right]$, respectively. Hence,

$$
f\left(u_{2}\right)>f\left(u_{1}\right), \quad f\left(u_{4}\right)>f\left(u_{3}\right), \quad g\left(u_{2}\right)<g\left(u_{1}\right) \quad \text { and } \quad g\left(u_{4}\right)<g\left(u_{3}\right) .
$$

The assumption that the pair $\{f, g\}$ is pairwise poorly convex on $[a, b]$, implies that for some positive numbers $h$ and $h^{\prime}$ we have

$$
f\left(u_{2}\right)-f\left(u_{1}\right) \leq h\left[f\left(u_{3}\right)-f\left(u_{2}\right)\right] \leq h h^{\prime}\left[f\left(u_{4}\right)-f\left(u_{3}\right)\right]
$$

and, similarly, the inequality $g\left(u_{2}\right)-g\left(u_{1}\right) \leq h h^{\prime}\left[g\left(u_{4}\right)-g\left(u_{3}\right)\right]$. Hence, in view of (22), we get $\frac{f\left(u_{2}\right)-f\left(u_{1}\right)}{f\left(u_{4}\right)-f\left(u_{3}\right)} \leq h h^{\prime} \leq \frac{g\left(u_{2}\right)-g\left(u_{1}\right)}{g\left(u_{4}\right)-g\left(u_{3}\right)}$, and thereby, $\frac{f\left(u_{2}\right)-f\left(u_{1}\right)}{g\left(u_{2}\right)-g\left(u_{1}\right)} \geq \frac{f\left(u_{4}\right)-f\left(u_{3}\right)}{g\left(u_{4}\right)-g\left(u_{3}\right)}$. But this, by the classical Cauchy's theorem, leads to the inequality, $\frac{f^{\prime}\left(\theta_{12}\right)}{g^{\prime}\left(\theta_{12}\right)} \geq \frac{f^{\prime}\left(\theta_{34}\right)}{g^{\prime}\left(\theta_{34}\right)}$ for some $\theta_{12} \in\left(u_{1}, u_{2}\right)$ and $\theta_{34} \in\left(u_{3}, u_{4}\right)$. Hence, taking into account that $\theta_{12} \rightarrow u_{1}$ as $u_{2} \rightarrow u_{1}$, and $\theta_{34} \rightarrow u_{4}$ as $u_{3} \rightarrow u_{4}$, we get $\frac{f^{\prime}\left(u_{1}\right)}{g^{\prime}\left(u_{1}\right)} \geq \frac{f^{\prime}\left(u_{4}\right)}{g^{\prime}\left(u_{4}\right)}$. In view of the arbitrarity of $u_{1}$ and $u_{4}$, it follows that the function $G(u)=f^{\prime}(u) / g^{\prime}(u)$ is nonincreasing on interval $\left(\tau^{f}, \tau_{g}\right)$.

$(\Leftarrow)$ Assume now that the function $G(u)=f^{\prime}(u) / g^{\prime}(u)$ is nonincreasing in $\left(\tau^{f}, \tau_{g}\right)$, and let us arbitrarily choose $\bar{u} \in Q_{g}^{f}$. Then Proposition 3 implies that

$$
f\left(u_{1}\right)<f\left(u_{2}\right)<f\left(u_{3}\right) \text { and } g\left(u_{1}\right)>g\left(u_{2}\right)>g\left(u_{3}\right) .
$$

Suppose now that inequality (13) does not hold. Then, in view of the above inequalities, we would have $\frac{f\left(u_{2}\right)-f\left(u_{1}\right)}{g\left(u_{2}\right)-g\left(u_{1}\right)}<\frac{f\left(u_{3}\right)-f\left(u_{2}\right)}{g\left(u_{3}\right)-g\left(u_{2}\right)}$. But this, by Cauchy's theorem, leads to the inequality $\frac{f^{\prime}\left(\theta_{12}\right)}{g^{\prime}\left(\theta_{12}\right)}<\frac{f^{\prime}\left(\theta_{23}\right)}{g^{\prime}\left(\theta_{23}\right)}$ for some $\theta_{12} \in\left(u_{1}, u_{2}\right)$ and $\theta_{23} \in\left(u_{2}, u_{3}\right)$, contradicting the assumption that function $G$ is nonincreasing in $\left(\tau^{f}, \tau_{g}\right)$. Therefore (13) holds, which by Proposition 6 completes the proof.

\section{On two properties of poorly convex functions}

In this section we use the theory from the previous section to present two main results describing some properties of poorly convex functions which are basic for the proofs of Theorems 1-5. We also give two examples illustrating the fact that the family of such functions are much richer than the class of convex functions.

In the first theorem we show that pairwise poor convexity of a finite family of functions can be remarkably strengthened. 
Theorem 6 Let a finite family $\mathcal{F}=\left\{f_{i}: i=1,2, \ldots, n\right\}$ of functions on $[a, b]$ be pairwise poorly convex. Then there is a positive function $T$ on $Q_{[a, b]}$ such that all the functions $f_{i}, i=1, \ldots, n$, are $T$-convex on $[a, b]$.

Proof Let us arbitrarily fix $\bar{u}=\left(u_{1}, u_{2}, u_{3}\right)$ in $Q_{[a, b]}$. It suffices to show that there is an $h>0$ such that

$$
f_{i}\left(u_{2}\right)-f_{i}\left(u_{1}\right) \leq h\left[f_{i}\left(u_{3}\right)-f_{i}\left(u_{2}\right)\right], \quad i=1,2, \ldots, n .
$$

Now, let $1 \leq k \leq n$. Since function $f_{k}$ is poorly convex, there is an $h_{k}>0$ such that

$$
f_{k}\left(u_{2}\right)-f_{k}\left(u_{1}\right) \leq h_{k}\left[f_{k}\left(u_{3}\right)-f_{k}\left(u_{2}\right)\right] .
$$

On the other hand, one can easily see that if the inequality (24) holds for two positive numbers $h_{k}=h_{k}^{\prime}$ and $h_{k}=h_{k}^{\prime \prime}$, then it also holds for every convex combination $h_{k}=\alpha h_{k}^{\prime}+(1-\alpha) h_{k}^{\prime \prime}, 0 \leq \alpha \leq 1$. Hence, it follows that all the sets

$$
A_{k}=\left\{h_{k}: h_{k}>0 \text { and inequality (24) holds }\right\}, k=1, \ldots, n,
$$

are nonempty and convex subsets of $R^{1}$. Now, taking into account the assumption about the pairwise poor convexity of family $\mathcal{F}$, we easily conclude that the class $\left\{A_{1}, A_{2}, \ldots, A_{n}\right\}$ of nonempty and convex subsets of $R^{1}$ has as property that $A_{j} \cap$ $A_{k} \neq \varnothing$ for any $1 \leq j, k \leq n, j \neq k$. Hence, by Helly's theorem (Eckhoff 1993), $\cap_{i=1}^{n} A_{i} \neq \emptyset$. But this implies that there is an $h>0$ such that (23) holds, completing the proof.

The last theorem we give in this section completes our considerations about poorly convex functions. It describes a nice property of families of such functions.

Theorem 7 Let $\mathcal{T}=\left\{f_{\alpha}: \alpha \in \mathcal{X}\right\}$ be a family of continuous functions on an interval $[a, b]$ such that every pair of functions in $\mathcal{T}$ is pairwise poorly convex. Let $\sup _{\alpha \in \mathcal{X}} f_{\alpha}(u)>0$ for each $u \in[a, b]$. Then there exist $\alpha, \beta \in \mathcal{X}$ and a vector $\left(\lambda_{1}, \lambda_{2}\right)$ with $\lambda_{1}, \lambda_{2} \geq 0$ and $\lambda_{1}+\lambda_{2}=1$, such that $\lambda_{1} f_{\alpha}(u)+\lambda_{2} f_{\beta}(u)>0$ for all $u \in[a, b]$.

Proof Let $A_{\alpha}=\left\{u \in[a, b]: f_{\alpha}(u)>0\right\}$ and $B_{\alpha}=\left\{u \in[a, b]: f_{\alpha}(u) \leq 0\right\}$. If $B_{\alpha^{\prime}}=\emptyset$ for some $\alpha^{\prime} \in \mathcal{X}$, then the theorem is satisfied by $\alpha=\alpha^{\prime}, \lambda_{1}=1, \lambda_{2}=0$ and arbitrary $\beta$. Therefore, for the rest of the proof we can assume that $B_{\alpha} \neq \varnothing$ for every $\alpha \in \mathcal{X}$

By assumption, every function $f_{\alpha}$ in $\mathcal{T}$ is continuous and poorly convex on $[a, b]$. Therefore, we can easily deduce with the help of Proposition 3 that for every $\alpha \in$ $\mathcal{X}, B_{\alpha}$ has the form of a nonempty closed interval, say $B_{\alpha}=\left[a_{\alpha}, b_{\alpha}\right]$, and thereby, $A_{\alpha}=\left[a, a_{\alpha}\right) \cup\left(b_{\alpha}, b\right]$.

The assumption of the theorem implies that $\cup_{\alpha \in \mathcal{X}} A_{\alpha}=[a, b]$. Since each $A_{\alpha}$ is an open set in $[a, b]$ (as a space), therefore there exists a finite cover $A_{\alpha_{1}}, A_{\alpha_{2}}, \ldots, A_{\alpha_{n}}$ of interval $[a, b]$ with minimal $n$. Consequently, for $i=1,2, \ldots, n$ we have

$$
f_{\alpha_{i}}(u) \leq 0 \quad \text { for } u \in\left[a_{\alpha_{i}}, b_{\alpha_{i}}\right]
$$


and

$$
f_{\alpha_{i}}(u)>0 \text { for } u \in\left[a, a_{\alpha_{i}}\right) \cup\left(b_{\alpha_{i}}, b\right] .
$$

Without loss of generality we can assume that $a_{\alpha_{1}} \leq a_{\alpha_{2}} \leq \ldots \leq a_{\alpha_{n}}$, and let $b_{\alpha_{q}}=$ $\min _{1 \leq i \leq n} b_{\alpha_{i}}$. The case $a_{\alpha_{n}} \leq b_{\alpha_{q}}$ cannot occur because it would imply that $f_{\alpha_{i}}(u) \leq 0$ for $u \in\left[a_{\alpha_{n}}, b_{\alpha_{q}}\right]$ and $i=1,2, \ldots, n$, contradicting the equality $\cup_{i=1}^{n} A_{\alpha_{i}}=[a, b]$. Therefore, $b_{\alpha_{q}}<a_{\alpha_{n}}$. We will show that the two functions $f_{\alpha}=f_{\alpha_{q}}$ and $f_{\beta}=f_{\alpha_{n}}$ satisfy the theorem with some positive $\lambda_{1}=\lambda^{*}$ and $\lambda_{2}=1-\lambda^{*}, 0<\lambda^{*}<1$.

One can easily deduce with the help of (25), (26) and Proposition 3 that continuous functions $f_{\alpha_{q}}$ and $f_{\alpha_{n}}$ are strictly increasing and strictly decreasing on interval $\left[b_{\alpha_{q}}, a_{\alpha_{n}}\right]$, respectively, and besides,

$$
f_{\alpha_{q}}\left(b_{\alpha_{q}}\right)=0 \quad \text { and } \quad f_{\alpha_{n}}\left(a_{\alpha_{n}}\right)=0
$$

Hence, one can easily deduce that there exists a $c$ such that

$$
\begin{aligned}
& b_{\alpha_{q}}<c<a_{\alpha_{n}}, f_{\alpha_{q}}(c)=f_{\alpha_{n}}(c)>0, \\
& f_{\alpha_{q}}(u)>0 \text { for } u \in\left(b_{\alpha_{q}}, c\right] \text { and } f_{\alpha_{n}}(u)>0 \text { for } u \in\left[b_{\alpha_{q}}, c\right],
\end{aligned}
$$

and

$$
f_{\alpha_{n}}\left(b_{\alpha_{q}}\right)>f_{\alpha_{n}}(c) \text { and } f_{\alpha_{q}}\left(b_{\alpha_{q}}\right)<f_{\alpha_{q}}(c)
$$

Consider now the continuous function $G(u, \lambda)=\lambda f_{\alpha_{q}}(u)+(1-\lambda) f_{\alpha_{n}}(u)$ defined on rectangle: $a \leq u \leq b, 0 \leq \lambda \leq 1$. By assumption, the pair of functions $\left\{f_{\alpha_{q}}, f_{\alpha_{n}}\right\}$ is pairwise poorly convex. Therefore there is a positive function $T$ on $Q_{[a, b]}$ such that functions $f_{\alpha_{q}}$ and $f_{\alpha_{n}}$ are $T$-convex. Hence, by statement (e) of Proposition 2, for any fixed $0 \leq \lambda \leq 1, G(u, \lambda)$ is a $T$-convex function of variable $u$, and thereby also poorly convex on $[a, b]$.

In view of inequalities (30), we have $G(c, 0)-G\left(b_{\alpha_{q}}, 0\right)=f_{\alpha_{n}}(c)-f_{\alpha_{n}}\left(b_{\alpha_{q}}\right)<0$ and $G(c, 1)-G\left(b_{\alpha_{q}}, 1\right)=f_{\alpha_{q}}(c)-f_{\alpha_{q}}\left(b_{\alpha_{q}}\right)>0$. Hence, by continuity of the function $F(\lambda)=G(c, \lambda)-G\left(b_{\alpha_{q}}, \lambda\right)$, there exists a $\lambda^{*}, 0<\lambda^{*}<1$, such that $F\left(\lambda^{*}\right)=0$. But this, with the help of (27), (28) and (29) implies that

$$
G\left(b_{\alpha_{q}}, \lambda^{*}\right)=G\left(c, \lambda^{*}\right)>0
$$

and

$$
G\left(u, \lambda^{*}\right)>0 \text { for } u \in\left[b_{\alpha_{q}}, c\right]
$$

Hence, in view of the inequality $b_{\alpha_{q}}<c$, Proposition 3 applied to function $G\left(u, \lambda^{*}\right)$ of variable $u$ easily implies that for all $u \in[a, b]$ we have $G\left(u, \lambda^{*}\right)>0$. Thus we have proved that $\lambda^{*} f_{\alpha_{q}}(u)+\left(1-\lambda^{*}\right) f_{\alpha_{n}}(u)>0$ for all $u \in[a, b]$, completing the proof. 
Remark 10 Theorem 7 obviously holds for any family $\mathcal{T}$ of convex continuous functions on interval $[a, b]$ because every such family is pairwise poorly convex (with respect to function $T(\bar{u})=\frac{u_{2}-u_{1}}{u_{3}-u_{2}}$ (see Remark 4 in Section 3)). Moreover, in the literature one can find the following more general result of Bohnenblust et al. (1950) (applied by them in game theory): If $\mathcal{T}$ is a family of convex continuous functions defined on a compact convex set $K$ in $R^{n}$ with the property that $\sup _{f \in \mathcal{T}} f(u)>0$ for each $u \in K$, then there are $n+1$ functions $f_{1}, \ldots, f_{n+1}$ in $\mathcal{T}$ and a vector $\left(\lambda_{1}, \ldots, \lambda_{n+1}\right)$ with nonnegative components such that $\sum_{i=1}^{n+1} \lambda_{i} f_{i}(u)>0$ for all $u \in K$. With this correlation, the natural question arises, if Theorem 7 can be generalized to families of poorly convex functions on $R^{n}$ ? Of course, this problem is closely related with the theory of poorly convex functions on $R^{n}$ which is not studied in this paper.

Pairwise poorly convex and concave families of functions are essential in the assumptions of Theorems 1-5. Therefore, we end this section with two examples presenting some of such families consisting of non-convex functions described with the help of very standard formulas. Proposition 7 appears to be a very useful tool in the analysis of such families. It is worth mentioning here that these examples show that the class of pairwise poorly convex functions is substantially richer than that of convex ones.

Example 3 Consider the family $\mathcal{F}=\left\{f_{\alpha}(x): \alpha \in[0, \sqrt{2}]\right\}$ of non-convex continuous functions with common domain $x \in[0, \sqrt{2}]$, where $f_{\alpha}(x)=-\exp \left\{-(x-\alpha)^{2}\right\}$. It can be easily verified that each function $f_{\alpha}(x)$ is strictly convex on interval $\left[c_{\alpha}, d_{\alpha}\right]=$ $[0, \sqrt{2}] \cap\left[\alpha-\frac{\sqrt{2}}{2}, \alpha+\frac{\sqrt{2}}{2}\right]$ and strictly concave on interval $\left[0, \alpha-\frac{\sqrt{2}}{2}\right]$ or on $\left[\alpha+\frac{\sqrt{2}}{2}, \sqrt{2}\right]$ if $\alpha \geq \frac{\sqrt{2}}{2}$ or $\alpha<\frac{\sqrt{2}}{2}$, respectively. Besides, each function $f_{\alpha}(x)$ is strictly decreasing in $[0, \alpha]$ and strictly increasing in $[\alpha, \sqrt{2}]$. Therefore, by Proposition $3, \mathcal{F}$ is a family of poorly convex functions on $[0, \sqrt{2}]$.

Now, let us arbitrarily choose two different functions $f(x)=f_{\alpha_{1}}(x)$ and $g(x)=$ $f_{\alpha_{2}}(x)$ from $\mathcal{F}$. So We assume that $0 \leq \alpha_{1}<\alpha_{2} \leq \sqrt{2}$. Obviously, $\tau^{f}=\alpha_{1}<$ $\alpha_{2}=\tau_{g}$. By direct computation we get that

$$
\left[f^{\prime}(x) / g^{\prime}(x)\right]^{\prime}=\frac{\alpha_{2}-\alpha_{1}}{\left(x-\alpha_{2}\right)^{2}}\left[2\left(x-\alpha_{1}\right)\left(\alpha_{2}-x\right)-1\right] e^{\left[\left(\alpha_{2}-\alpha_{1}\right)\left(\alpha_{2}+\alpha_{1}\right)-2 x\right]}
$$

But $2\left(x-\alpha_{1}\right)\left(\alpha_{2}-x\right)-1 \leq 0$ for $x \in\left(\alpha_{1}, \alpha_{2}\right)$ (because $\left.\left(\alpha_{1}, \alpha_{2}\right) \subset[0, \sqrt{2}]\right)$. Therefore this implies that $\left[f^{\prime}(x) / g^{\prime}(x)\right]^{\prime} \leq 0$ for $x \in\left(\alpha_{1}, \alpha_{2}\right)$, and thereby function $G(x)=f^{\prime}(x) / g^{\prime}(x)$ is nonincreasing in interval $\left(\tau^{f}, \tau_{g}\right)$. Hence, by Proposition 7 , every pair of functions on family $\mathcal{F}$ is pairwise poorly convex.

Example 4 Consider the family $\mathcal{U}=\left\{f_{\alpha \beta \gamma}(x): \alpha \in R, \beta<0, \gamma \in\left[-\frac{\pi}{2}, \frac{\pi}{2}\right]\right\}$ of non-convex continuous functions with common domain $-\frac{\pi}{2} \leq x \leq \frac{\pi}{2}$, where $f_{\alpha \beta \gamma}(x)=\alpha+\beta \sin (x-\gamma)$. The fact that each function in family $\mathcal{U}$ is poorly convex easily follows from Proposition 3 .

Let us arbitrarily choose two different functions $f(x)=f_{\alpha_{1} \beta_{1} \gamma_{1}}(x)$ and $g(x)=$ $f_{\alpha_{2} \beta_{2} \gamma_{2}}(x)$ from $\mathcal{U}$. We easily deduce that $\tau_{f}=\tau^{f}=\gamma_{1}-\frac{\pi}{2}$ and $\tau_{g}=\tau^{g}=\gamma_{2}-\frac{\pi}{2}$. 
When $\gamma_{1}=\gamma_{2}$, the pair of functions $f(x)$ and $g(x)$ are pairwise poorly convex because of Proposition 5. For the second case we can assume that $-\frac{\pi}{2} \leq \gamma_{1}<\gamma_{2} \leq \frac{\pi}{2}$, whence $\tau^{f}<\tau_{g}$. Then we easily get

$$
\left[f^{\prime}(x) / g^{\prime}(x)\right]^{\prime}=-\frac{\beta_{1} \sin \left(\gamma_{2}-\gamma_{1}\right)}{\beta_{2} \cos ^{2}\left(x-\gamma_{2}\right)} .
$$

Hence, $\left[f^{\prime}(x) / g^{\prime}(x)\right]^{\prime} \leq 0$ for $x \in\left(\tau^{f}, \tau_{g}\right)$, because of $0<\gamma_{2}-\gamma_{1} \leq \pi$. Therefore, by Proposition 7, every pair of functions in family $\mathcal{U}$ is pairwise poorly convex.

\section{Proofs of the main theorems}

In this section we prove our main Theorems 1-5 from Sect. 4.

Proof of Theorem 1 We construct two matrices $A^{n}$ and $B^{n}$ of dimension $n \times n$ with their elements denoted by $a_{i j}^{n}$ and $b_{i j}^{n}$, and defined by the following: $a_{i j}^{n}=F_{1}\left(\frac{i}{n}, \frac{j}{n}\right)$ and $b_{i j}^{n}=F_{2}\left(\frac{i}{n}, \frac{j}{n}\right), i, j=1,2, \ldots, n$. Further, let $\Gamma\left(A^{n}, B^{n}\right)$ be the bimatrix game with payoff matrices $A^{n}$ and $B^{n}$ for Players 1 and 2 , respectively.

Let $n$ be a natural number and consider the finite family $F=\left\{f_{i}(u): i=1, \ldots, n\right\}$ of functions on $[0,1]$, where $f_{i}(u)=F_{1}\left(u, \frac{i}{n}\right)$ for $1 \leq i \leq n$ and $0 \leq u \leq 1$. By assumption, family $F$ is pairwise poorly concave. Therefore Theorem 6 and statement (a) of Proposition 2 imply that there is a positive function $H\left(u_{1}, u_{2}, u_{3}\right)$ on $Q_{[0,1]}$ such that for all $0 \leq u_{1}<u_{2}<u_{3} \leq 1$,

$F_{1}\left(u_{2}, \frac{i}{n}\right)-F_{1}\left(u_{1}, \frac{i}{n}\right) \geq H\left(u_{1}, u_{2}, u_{3}\right)\left[F_{1}\left(u_{3}, \frac{i}{n}\right)-F_{1}\left(u_{2}, \frac{i}{n}\right)\right] \quad$ for $i=1, \ldots, n$.

But this implies that for the positive constants $h_{i}=H\left(\frac{i}{n}, \frac{i+1}{n}, \frac{i+2}{n}\right), 1 \leq i \leq n-2$, and for all $j, 1 \leq j \leq n$, the inequalities hold: $a_{i+1, j}^{n}-a_{i j}^{n} \geq h_{i}\left(a_{i+2, j}^{n}-a_{i+1, j}^{n}\right)$ for $i=1,2, \ldots, n-2$. Hence, we can easily see that there is a sequence $\theta_{1}, \theta_{2}, \ldots, \theta_{n-1}$ of positive numbers such that for all $j, 1 \leq j \leq n$,

$$
\theta_{1}\left(a_{2 j}^{n}-a_{1 j}^{n}\right) \geq \theta_{2}\left(a_{3 j}^{n}-a_{2 j}^{n}\right) \geq \ldots \geq \theta_{n-1}\left(a_{n j}^{n}-a_{n-1, j}^{n}\right) .
$$

Therefore the bimatrix game $\Gamma\left(A^{n}, B^{n}\right)$ is column-concave [see Definition 1 and Theorem 4 in Połowczuk (2006)], and consequently, it follows [by Theorem 7 in Połowczuk (2006)] that game $\Gamma\left(A^{n}, B^{n}\right)$ has a Nash equilibrium $\left(\mu_{n}, v_{n}\right)$ of the form $\mu_{n}=\lambda_{n} \delta_{s_{n}}+\left(1-\lambda_{n}\right) \delta_{s_{n}+1}$ and $v_{n}=\gamma_{n} \delta_{r_{n}}+\left(1-\gamma_{n}\right) \delta_{u_{n}}$ for some reals $0 \leq \lambda_{n}, \gamma_{n} \leq 1$ and naturals $1 \leq s_{n}<n, 1 \leq r_{n}, u_{n} \leq n$. Therefore, by the Nash equilibrium inequality,

$$
\begin{aligned}
& \lambda_{n} \gamma_{n} a_{s_{n}, r_{n}}+\left(1-\lambda_{n}\right) \gamma_{n} a_{s_{n}+1, r_{n}}+\lambda_{n}\left(1-\gamma_{n}\right) a_{s_{n}, u_{n}}+\left(1-\lambda_{n}\right)\left(1-\gamma_{n}\right) a_{s_{n}+1, u_{n}} \\
& \quad \geq \gamma_{n} a_{i, r_{n}}+\left(1-\gamma_{n}\right) a_{i, u_{n}} \text { for } i=1, \ldots, n,
\end{aligned}
$$

and 


$$
\begin{aligned}
& \lambda_{n} \gamma_{n} b_{s_{n}, r_{n}}+\left(1-\lambda_{n}\right) \gamma_{n} b_{s_{n}+1, r_{n}}+\lambda_{n}\left(1-\gamma_{n}\right) b_{s_{n}, u_{n}}+\left(1-\lambda_{n}\right)\left(1-\gamma_{n}\right) b_{s_{n}+1, u_{n}} \\
& \geq \lambda_{n} b_{s_{n}, j}+\left(1-\lambda_{n}\right) b_{s_{n}+1, j} \text { for } j=1, \ldots, n .
\end{aligned}
$$

But these inequalities are equivalent to

$$
\begin{aligned}
& \lambda_{n} \gamma_{n} F_{1}\left(\frac{s_{n}}{n}, \frac{r_{n}}{n}\right)+\left(1-\lambda_{n}\right) \gamma_{n} F_{1}\left(\frac{s_{n}+1}{n}, \frac{r_{n}}{n}\right)+\lambda_{n}\left(1-\gamma_{n}\right) F_{1}\left(\frac{s_{n}}{n}, \frac{u_{n}}{n}\right) \\
& +\left(1-\lambda_{n}\right)\left(1-\gamma_{n}\right) F_{1}\left(\frac{s_{n}+1}{n}, \frac{u_{n}}{n}\right) \geq \gamma_{n} F_{1}\left(\frac{i}{n}, \frac{r_{n}}{n}\right) \\
& \quad+\left(1-\gamma_{n}\right) F_{1}\left(\frac{i}{n}, \frac{u_{n}}{n}\right) \quad \text { for } i=1, \ldots, n,
\end{aligned}
$$

and

$$
\begin{aligned}
& \lambda_{n} \gamma_{n} F_{2}\left(\frac{s_{n}}{n}, \frac{r_{n}}{n}\right)+\left(1-\lambda_{n}\right) \gamma_{n} F_{2}\left(\frac{s_{n}+1}{n}, \frac{r_{n}}{n}\right)+\lambda_{n}\left(1-\gamma_{n}\right) F_{2}\left(\frac{s_{n}}{n}, \frac{u_{n}}{n}\right) \\
& +\left(1-\lambda_{n}\right)\left(1-\gamma_{n}\right) F_{2}\left(\frac{s_{n}+1}{n}, \frac{u_{n}}{n}\right) \geq \lambda_{n} F_{2}\left(\frac{s_{n}}{n}, \frac{j}{n}\right) \\
& \quad+\left(1-\lambda_{n}\right) F_{2}\left(\frac{s_{n}+1}{n}, \frac{j}{n}\right) \quad \text { for } i=1, \ldots, n .
\end{aligned}
$$

Now, letting $n \rightarrow \infty$, we can obviously choose a subsequence $n^{\prime} \rightarrow \infty$ to have the convergence $\lambda_{n^{\prime}} \rightarrow \alpha, \gamma_{n^{\prime}} \rightarrow \beta, s_{n^{\prime}} / n^{\prime} \rightarrow a, r_{n^{\prime}} / n^{\prime} \rightarrow c$ and $u_{n^{\prime}} / n^{\prime} \rightarrow d$ for some $0 \leq \alpha, \beta, a, c, d \leq 1$. Therefore also $\left(s_{n^{\prime}}+1\right) / n^{\prime} \rightarrow a$.

Further, let us arbitrarily choose $0 \leq x, y \leq 1$. Of course, there are sequences $i^{\prime}, j^{\prime} \rightarrow \infty$ such that $i^{\prime} / n^{\prime} \rightarrow x$ and $j^{\prime} / n^{\prime} \rightarrow y$.

Hence, taking into account the continuity of functions $F_{1}$ and $F_{2}$, the last two inequalities imply (after taking $n=n^{\prime} \rightarrow \infty$ ) the following: for any $0 \leq x, y \leq 1$

$$
\beta F_{1}(a, c)+(1-\beta) F_{1}(a, d) \geq \beta F_{1}(x, c)+(1-\beta) F_{1}(x, d)
$$

and

$$
\beta F_{2}(a, c)+(1-\beta) F_{2}(a, d) \geq F_{2}(a, y) .
$$

After defining two strategies $\mu^{*}=\delta_{a}$ and $v^{*}=\beta \delta_{c}+(1-\beta) \delta_{d}$ for Players 1 and 2 in game $\Gamma$, respectively, the last inequalities can be equivalently rewritten as

$$
F_{1}\left(\mu^{*}, v^{*}\right) \geq F_{1}\left(x, v^{*}\right) \quad \text { and } \quad F_{2}\left(\mu^{*}, v^{*}\right) \geq F_{2}\left(\mu^{*}, y\right), \quad 0 \leq x, y \leq 1 .
$$

Thus $\left(\mu^{*}, v^{*}\right)$ is a Nash equilibrium in game $\Gamma$, which ends the proof of Theorem 1. 
Proof of Theorem 2 Let us define an auxiliary non-zero-sum two-person game $\tilde{\Gamma}$ on the unit square with payoff functions $\tilde{F}_{1}$ and $\tilde{F}_{2}$ of the form

$$
\tilde{F}_{1}\left(p, x_{2}\right)=p F_{1}\left(0, x_{2}\right)+(1-p) F_{1}\left(1, x_{2}\right), \quad 0 \leq p, x_{2} \leq 1,
$$

and

$$
\tilde{F}_{2}\left(p, x_{2}\right)=p F_{2}\left(0, x_{2}\right)+(1-p) F_{2}\left(1, x_{2}\right) . \quad 0 \leq p, x_{2} \leq 1 .
$$

By assumption, $\tilde{F}_{1}\left(p, x_{2}\right)$ and $\tilde{F}_{2}\left(p, x_{2}\right)$ are continuous functions on $[0,1]^{2}$. Additionally, function $\tilde{F}_{1}\left(p, x_{2}\right)$ is linear in variable $p$, and thereby concave in $p$. Hence, the family of functions $\left\{\tilde{F}_{1}\left(\cdot, x_{2}\right): 0 \leq x_{2} \leq 1\right\}$ is pairwise poorly concave (as stated in Remark 4 in Sect. 3). Therefore game $\tilde{\Gamma}$ satisfies the assumptions of Theorem 1 and consequently, there are $0 \leq \alpha, \beta, c, d \leq 1$ such that the pair of strategies $\mu_{1}^{0}=\delta_{\alpha}$ and $\mu_{2}^{0}=\beta \delta_{c}+(1-\beta) \delta_{d}$ creates a Nash equilibrium in game $\tilde{\Gamma}$. This implies the following inequalities:

$\tilde{F}_{1}\left(x_{1}, \mu_{2}^{0}\right) \leq \tilde{F}_{1}\left(\mu_{1}^{0}, \mu_{2}^{0}\right) \quad$ and $\quad \tilde{F}_{2}\left(\mu_{1}^{0}, x_{2}\right) \leq \tilde{F}_{2}\left(\mu_{1}^{0}, \mu_{2}^{0}\right) \quad$ for $\quad 0 \leq x_{1}, x_{2} \leq 1$.

We will show that the pair $\left(\mu_{1}^{*}, \mu_{2}^{*}\right)$ with $\mu_{1}^{*}=\alpha \delta_{0}+(1-\alpha) \delta_{1}$ and $\mu_{2}^{*}=\mu_{2}^{0}$ is a Nash equilibrium in game $\Gamma$.

First notice that

$$
\tilde{F}_{i}\left(\mu_{1}^{0}, \mu_{2}^{0}\right)=F_{i}\left(\mu_{1}^{*}, \mu_{2}^{*}\right), \quad i=1,2
$$

By assumption, the family of functions $\left\{F_{1}\left(\cdot, x_{2}\right): 0 \leq x_{2} \leq 1\right\}$ is pairwise poorly convex. Hence, for every $0<x_{1}<1$ there is a positive number $h\left(x_{1}\right)$ such that

$$
F_{1}\left(x_{1}, c\right)-F_{1}(0, c) \leq h\left(x_{1}\right)\left[F_{1}(1, c)-F_{1}\left(x_{1}, c\right)\right]
$$

and

$$
F_{1}\left(x_{1}, d\right)-F_{1}(0, d) \leq h\left(x_{1}\right)\left[F_{1}(1, d)-F_{1}\left(x_{1}, d\right)\right] .
$$

Using (33) and the definition of $\tilde{F}_{1}$, for $0<x_{1}<1$, we have

$$
F_{1}\left(x_{1}, c\right) \leq \frac{1}{1+h\left(x_{1}\right)} F_{1}(0, c)+\frac{h\left(x_{1}\right)}{1+h\left(x_{1}\right)} F_{1}(1, c)=\tilde{F}_{1}\left(\frac{1}{1+h\left(x_{1}\right)}, c\right) .
$$

Besides, $F_{1}(0, c)=\tilde{F}_{1}(1, c)$ and $F_{1}(1, c)=\tilde{F}_{1}(0, c)$. Therefore, for any $0 \leq x_{1} \leq 1$ there is a number $p_{x_{1}} \in[0,1]$ such that

$$
F_{1}\left(x_{1}, c\right) \leq \tilde{F}_{1}\left(p_{x_{1}}, c\right) \text { for } \quad 0 \leq x_{1} \leq 1 .
$$

Exactly in the same way, using (34), we also get 


$$
F_{1}\left(x_{1}, d\right) \leq \tilde{F}_{1}\left(p_{x_{1}}, d\right) \quad \text { for } \quad 0 \leq x_{1} \leq 1
$$

But the last two inequalities imply that $F_{1}\left(x_{1}, \mu_{2}^{0}\right) \leq \tilde{F}_{1}\left(p_{x_{1}}, \mu_{2}^{0}\right)$ for $0 \leq x_{1} \leq 1$.

Now, using this inequality, (31), (32) and the equality $\mu_{2}^{*}=\mu_{2}^{0}$, we can conclude as follows:

$$
F_{1}\left(x_{1}, \mu_{2}^{*}\right)=F_{1}\left(x_{1}, \mu_{2}^{0}\right) \leq \tilde{F}_{1}\left(p_{x_{1}}, \mu_{2}^{0}\right) \leq \tilde{F}_{1}\left(\mu_{1}^{0}, \mu_{2}^{0}\right)=F_{1}\left(\mu_{1}^{*}, \mu_{2}^{*}\right)
$$

Consequently,

$$
F_{1}\left(x_{1}, \mu_{2}^{*}\right) \leq F_{1}\left(\mu_{1}^{*}, \mu_{2}^{*}\right) \text { for } 0 \leq x_{1} \leq 1 .
$$

Similarly, using (31), (32) and the definition of strategy $\mu_{1}^{*}$, for all $x_{2} \in[0,1]$ we have

$$
\begin{aligned}
F_{2}\left(\mu_{1}^{*}, x_{2}\right) & =\alpha F_{2}\left(0, x_{2}\right)+(1-\alpha) F_{2}\left(1, x_{2}\right)=\tilde{F}_{2}\left(\mu_{1}^{0}, x_{2}\right) \leq \tilde{F}_{2}\left(\mu_{1}^{0}, \mu_{2}^{0}\right) \\
& =F_{2}\left(\mu_{1}^{*}, \mu_{2}^{*}\right)
\end{aligned}
$$

Therefore, $F_{2}\left(\mu_{1}^{*}, x_{2}\right) \leq F_{2}\left(\mu_{1}^{*}, \mu_{2}^{*}\right)$ for $0 \leq x_{2} \leq 1$, which together with (35) completes the proof of Theorem 2 .

Proof of Theorem 3 Assume that $(\mu, v)$ is a Nash equilibrium in game $\Gamma$. Let $x_{1} \in$ $[0,1]$ and suppose that $F_{1}\left(\mu, x_{2}^{\prime}\right)<F_{1}\left(x_{1}, x_{2}^{\prime}\right)$ for all $x_{2}^{\prime} \in \operatorname{supp}(v)$. But then this inequality would imply that $F_{1}(\mu, v)<F_{1}\left(x_{1}, v\right)$ which contradicts the fact that $(\mu, v)$ is a Nash equilibrium.

Let us fix $\varepsilon>0$. Therefore for all $x_{1} \in[0,1]$

$$
\sup _{x_{2} \in \operatorname{supp}(v)}\left[F_{1}\left(\mu, x_{2}\right)-F_{1}\left(x_{1}, x_{2}\right)+\varepsilon\right]>0 .
$$

Let us denote $G_{x_{2}}\left(x_{1}\right)=F_{1}\left(\mu, x_{2}\right)-F_{1}\left(x_{1}, x_{2}\right)+\varepsilon$. By assumption, the family of functions $\left\{F_{1}\left(\cdot, x_{2}\right): 0 \leq x_{2} \leq 1\right\}$ is pairwise poorly concave. Hence, we can easily state with the help of statement (a) of Proposition 2 that the family of functions $\left\{G_{x_{2}}\left(x_{1}\right): x_{2} \in \operatorname{supp}(v)\right\}$ of variable $x_{1}$ is pairwise poorly convex on $[0,1]$ and satisfies the assumptions of Theorem 7. Therefore, there are $c, d \in \operatorname{supp}(v)$ and a probability vector $(\beta, 1-\beta)$ such that for all $x_{1} \in[0,1]$ we have $\beta G_{c}\left(x_{1}\right)+(1-$ $\beta) G_{d}\left(x_{1}\right)>0$. But this, in view of the arbitrarity of $\varepsilon$, implies that for each $x_{1} \in[0,1]$

$$
\beta F_{1}(\mu, c)+(1-\beta) F_{1}(\mu, d) \geq \beta F_{1}\left(x_{1}, c\right)+(1-\beta) F_{1}\left(x_{1}, d\right),
$$

or equivalently,

$$
F_{1}\left(\mu, \beta \delta_{c}+(1-\beta) \delta_{d}\right) \geq F_{1}\left(x_{1}, \beta \delta_{c}+(1-\beta) \delta_{d}\right) .
$$

On the other hand, since $(\mu, v)$ is a Nash equilibrium,

$$
F_{2}(\mu, v) \geq F_{2}\left(\mu, x_{2}\right) \text { for } \quad 0 \leq x_{2} \leq 1
$$


Besides, $c, d \in \operatorname{supp}(v)$ and function $F_{2}\left(x_{1}, x_{2}\right)$ is continuous on $[0,1]^{2}$. This and inequality (37) imply that $F_{2}(\mu, c)=F_{2}(\mu, d)=F_{2}(\mu, v)$, and consequently

$$
F_{2}(\mu, \nu)=F_{2}\left(\mu, \beta \delta_{c}+(1-\beta) \delta_{d}\right)
$$

Now, taking into account this equality, (37) and (36), we easily deduce that the pair $\left(\mu, \beta \delta_{c}+(1-\beta) \delta_{d}\right)$ is also a Nash equilibrium in game $\Gamma$, and it satisfies Theorem 3. Thus the proof has been completed.

Proof of Theorem 4 It is an immediate consequence of Theorem 3.

Proof of Theorem 5 Let us define an auxiliary non-zero-sum two-person game $\hat{\Gamma}$ on the unit square with payoff functions $\hat{F}_{1}(p, q)$ and $\hat{F}_{2}(p, q), 0 \leq p, q \leq 1$, of the form

$$
\begin{aligned}
\hat{F}_{i}(p, q)= & p q F_{i}(0,0)+(1-p) q F_{i}(1,0)+p(1-q) F_{i}(0,1) \\
& +(1-p)(1-q) F_{i}(1,1)
\end{aligned}
$$

for $i=1,2$.

We easily see that payoff functions $\hat{F}_{1}(p, q)$ and $\hat{F}_{2}(p, q)$ are continuous on $[0,1]^{2}$ and linear in each variable. Therefore they satisfy the assumptions of Theorem $\mathrm{A}$ in Sect. 2. Consequently, there are $0 \leq \alpha, \beta \leq 1$ such that the pair of strategies $\mu_{1}^{0}=\delta_{\alpha}$ and $\mu_{2}^{0}=\delta_{\beta}$ is a Nash equilibrium in game $\hat{\Gamma}$. We will show that the pair $\left(\mu_{1}^{*}, \mu_{2}^{*}\right)$ with $\mu_{1}^{*}=\alpha \delta_{0}+(1-\alpha) \delta_{1}$ and $\mu_{1}^{*}=\beta \delta_{0}+(1-\beta) \delta_{1}$ is a Nash equilibrium in game $\Gamma$.

First notice that

$$
\hat{F}_{i}\left(\mu_{1}^{0}, \mu_{2}^{0}\right)=F_{i}\left(\mu_{1}^{*}, \mu_{2}^{*}\right), \quad i=1,2
$$

By assumption, the family of functions $\left\{F_{1}\left(\cdot, x_{2}\right): 0 \leq x_{2} \leq 1\right\}$ is pairwise poorly convex. Now, using this and (38), one can easily see that after changing $\tilde{F}_{1}$ with $\hat{F}_{1}$, the reasoning given between formulae (33) and (35) can be repeated with $c=0, d=1$ to get that $F_{1}\left(x_{1}, \mu_{2}^{*}\right) \leq F_{1}\left(\mu_{1}^{*}, \mu_{2}^{*}\right)$ for $0 \leq x_{1} \leq 1$. On the other hand, since also the family of functions $\left\{F_{2}\left(x_{1}, \cdot\right): 0 \leq x_{1} \leq 1\right\}$ is pairwise poorly convex, by the same way $F_{2}\left(\mu_{1}^{*}, x_{2}\right) \leq F_{2}\left(\mu_{1}^{*}, \mu_{2}^{*}\right)$ for $0 \leq x_{2} \leq 1$. Therefore the pair $\left(\mu_{1}^{*}, \mu_{2}^{*}\right)$ is a Nash equilibrium in game $\Gamma$, which completes the proof.

Acknowledgments This research was supported by GRANT 342711 of Wrocław University of Technology. The author thanks two anonymous referees for their useful comments, suggestions and inspiring questions, which have enabled the author to improve the paper.

Open Access This article is distributed under the terms of the Creative Commons Attribution License which permits any use, distribution, and reproduction in any medium, provided the original author(s) and the source are credited. 


\section{References}

Bich P (2009) Existence of pure Nash equilibria in discontinuous and quasiconcave games. Int J Game Theory 38:395-410

Bohnenblust HF, Karlin S, Shapley LS (1950) Games with continuous convex payoff. In: Kuhn HW, Tucker AW (eds) Annals mathematics studies, vol 24. Princeton University Press, Princeton, pp 181-192

Carmona G (2009) An existence result for discontinuous games. J Econ Theory 144(3):1333-1340

Carmona G (2010) Polytopes and existence of approximate equilibria in discontinuous games. Games Econ Behav 68:381-398

Eckhoff J (1993) Helly, Radon, and Carathodory type theorems. Handbook of convex geometry, A, B. North-Holland, Amsterdam, pp 389-448

Glicksberg LA (1952) A further generalization of the Kakutani's fixed point theorem with application to Nash equilibrium points. Proc Am Math Soc 38:170-174

McClendon JP (1986) Existence of solutions of games with some non-convexity. Int J Game Theory 15 : 155-162

Nash J (1951) Non-cooperative games. Ann Math 54:286-295

Parthasarathy T, Raghavan TES (1975) Equilibria of continuous two-person games. Pac J Math 57:265-270

Połowczuk W (2006) On almost-pure Nash equilibria in bimatrix games with convexity properties. Appl Math 33:71-84

Połowczuk W (2003) Pure Nash equilibria in finite two-person non-zero-sum games. Int J Game Theory 32:229-240

Połowczuk W, Więcek P, Radzik T (2007) On the existence of almost-pure strategy Nash equilibrium in $n$-person finite games. Math Methods Oper Res 65:141-152

Radzik T, Ravindran G (1989) On two counterexamples of non-cooperative games without Nash equilibrium. Sankyā. Indian J Stat A 51:236-240

Radzik T (1991) Pure-strategy $\varepsilon$-Nash equilibrium in two-person non-zero-sum games. Games Econ Behavr 3:356-367

Radzik T (1993) Nash equilibria of discontinuous non-zero-sum two-person games. Int J Game Theory 21:429-437

Radzik T (2000) Characterization of optimal strategies in matrix games with convexity properties. Int J Game Theory 29:211-227 